\title{
Synergistic enhancement of cancer therapy using a combination of docetaxel and photothermal ablation induced by single-walled carbon nanotubes
}

\author{
This article was published in the following Dove Press journal: \\ International Journal of Nanomedicine \\ 28 October 2011 \\ Number of times this article has been viewed
}

\author{
Lei Wang' \\ Mingyue Zhang' \\ Nan Zhang' \\ Jinjin Shi' \\ Hongling Zhang' \\ Min $\mathrm{Li}^{\prime}$ \\ Chao Lu ${ }^{2}$ \\ Zhenzhong Zhang' \\ 'School of Pharmaceutical Sciences, \\ Zhengzhou University, Zhengzhou, \\ People's Republic of China; ${ }^{2}$ University \\ of Maryland, College Park, MD, USA
}

Correspondence: Zhenzhong Zhang School of Pharmaceutical Sciences, Zhengzhou University, Zhengzhou, 100 Kexue Avenue, 450000, People's Republic of China Tel $+8637 \mid 67781910$ Fax +86 37। 67781908 Email zhangzz08@I26.com
Background: Single-walled carbon nanotubes (SWNT) are poorly soluble in water, so their applications are limited. Therefore, aqueous solutions of SWNT, designed by noncovalent functionalization and without toxicity, are required for biomedical applications.

Methods: In this study, we conjugated docetaxel with SWNT via $\pi$ - $\pi$ accumulation and used a surfactant to functionalize SWNT noncovalently. The SWNT were then conjugated with docetaxel (DTX-SWNT) and linked with NGR (Asn-Gly-Arg) peptide, which targets tumor angiogenesis, to obtain a water-soluble and tumor-targeting SWNT-NGR-DTX drug delivery system.

Results: SWNT-NGR-DTX showed higher efficacy than docetaxel in suppressing tumor growth in a cultured PC3 cell line in vitro and in a murine S180 cancer model. Tumor volumes in the $\mathrm{S} 180$ mouse model decreased considerably under near-infrared radiation compared with the control group.

Conclusion: The SWNT-NGR-DTX drug delivery system may be promising for high treatment efficacy with minimal side effects in future cancer therapy.

Keywords: single-walled carbon nanotubes, docetaxel, NGR peptide, tumor-targeting, near-infrared radiation

\section{Introduction}

Due to the unique physical and chemical properties of carbon nanotubes, they are considered to be potential biomedical materials for delivery of biological macromolecules and chemicals into cells and living systems for the purposes of disease diagnosis and therapy. ${ }^{1-3}$ According to the size of single-walled nanotubes (SWNT) and their uptake by cells, targeted delivery of SWNT-borne pharmaceuticals at the cellular level could be enhanced, and the discomfort associated with current intrusive techniques could be minimized. SWNT is a unique one-dimensional material, and has been explored as a novel drug delivery vehicle in vitro. ${ }^{4,5}$ SWNT can effectively shuttle various biomolecules including drugs, peptides, proteins, plasmid DNA, and small interfering RNA into cells via endocytosis. ${ }^{6-9}$ SWNT are not soluble in aqueous solution, which limits their application. However, SWNT with particular design features are able to enter cells without toxicity, giving this nanomaterial wide biomedical applications. ${ }^{10-12}$

Angiogenic tumor vessels are important elements for tumor growth and metastasis. They are essential for transporting metabolically essential materials to and from tumor cells, and also provide a route for dissemination of tumor cells to distal sites. The Asn-Gly-Arg (NGR) peptide motif has been used as a targeting head for target drugs and drug-containing liposomes to CD13, the tumor vascular antigen, ${ }^{13,14}$ resulting 
in improved biodistribution and more effective tumor therapy. ${ }^{15,16}$ Peptides containing NGR have shown high efficiency in targeted cells and tissues. ${ }^{17,18}$

Docetaxel (N-debenzoyl-N-tert-butoxy-carbonyl-10deacetyl) is a new class of taxane, which has achieved higher patient response rates and fewer side effects compared with paclitaxel in the treatment of a wide spectrum of cancers, including breast, ovarian, small and nonsmall cell lung, and head/neck cancer. ${ }^{19-22}$ Both paclitaxel and docetaxel act by binding to microtubules and inhibiting microtubule depolymerization to free tubulin. As a result, the equilibrium within the microtubule system is disrupted, leading to mitotic arrest in the G2/M phase of the cell cycle and ultimately to cell death. ${ }^{23}$ In this study, docetaxel was used as a model anticancer agent.

Biological systems are known to be highly transparent under 700-1100 nm near-infrared light. The strong optical absorbance of SWNT can be used for photothermal therapy to afford multifunctional biological nanotube transporters. ${ }^{24-28}$ Continuous near-infrared irradiation can cause cell death in vitro because of excessive local heating of the SWNT. ${ }^{29}$

In this study, we developed a SWNT-NGR delivery system conjugated with docetaxel (SWNT-NGR-DTX).
In this delivery system, docetaxel was attached through $\pi-\pi$ accumulation to SWNT and dispersed in water with PVPk30 and 1,2-distearoyl-sn-glycero-3-phosphoethanolamine-N-[methoxy(polyethylene glycol)-2000] (DSPEPEG2000)-maleimide (DPM). Because the maleimide group at the end of DPM on the surface of SWNT can react with the double bond in the sulfhydryl groups, the maleimide was reacted with cysteine in $\mathrm{CNGRCK}_{2} \mathrm{HK}_{3} \mathrm{HK}_{11}$ (C containing sulfhydryl groups) and connected covalently. This delivery system targeted as tumor cells as shown in Figure 1. It was hypothesized that the docetaxel attached to SWNT would be released from the SWNT-NGR-DTX delivery system in a sustained fashion, and the ability of docetaxel to kill tumor cells would be enhanced through near-infrared irradiationmediated tumor destruction by the photothermal effect on the carbon nanotubes.

\section{Materials and methods}

SWNT produced by chemical vapor deposition (purity $>90 \%$ ) were purchased from Chengdu Organic Chemicals Co Ltd (Chengdu, China). A PC3 human prostate cancer cell line and S180 mouse ascites tumor cells were obtained from the Chinese Academy of Sciences Cell Bank. NGR peptides (CNGRCK $\mathrm{HK}_{3} \mathrm{HK}_{11}$ ) were compounded by Shanghai
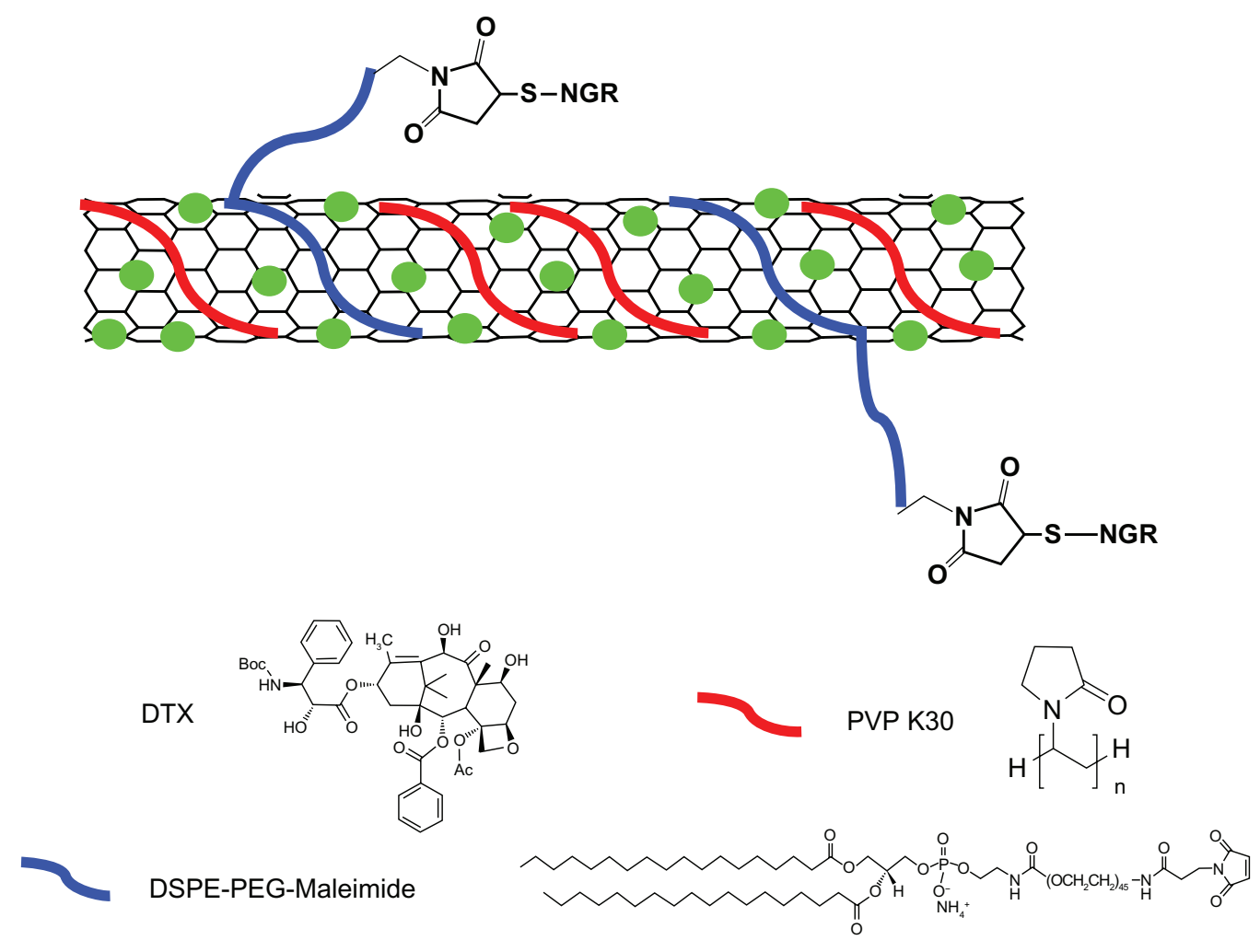

Figure I Docetaxel delivery system using noncovalent functionalization of carbon nanotubes.

Abbreviations: DSPE-PEG, I,2-distearoyl-sn-glycero-3-phosphoethanolamine-N-[methoxy(polyethylene glycol)-2000]; NGR, (Asn-Gly-Arg) peptide; DTX, docetaxel. 
Botai Biotechnology Co Ltd (Shanghai, China). Docetaxel was purchased from Beijing YiHe Biotechnology Co Ltd, DPM and other reagents were all purchased from Sigma (St Louis, MO). Experimental animals were provided by the Henan Laboratory Animal Center.

\section{Preparation of SWNT-DTX}

SWNT $5 \mathrm{mg}$ was mixed with docetaxel $20 \mathrm{mg}$ in anhydrous ethanol $0.5 \mathrm{~mL}$ using an ultrasonic bath for about 15 minutes while dropwise phosphate-buffered solution $3 \mathrm{~mL}$ was added. The mixture was ultrasonicated using an ultrasonic probe (400 W, 10 times). The suspension was centrifuged at 10,000 rpm for 15 minutes until the SWNT were fully precipitated, and the supernatants were discarded. The remaining solids were thoroughly rinsed with anhydrous ethanol and deionized water to remove excess docetaxel.

\section{Conjugation of DPM to SWNT-DTX}

The SWNT-DTX were resuspended in an aqueous solution of PVPk30 $10 \mathrm{mg} / \mathrm{mL}$ and DPM $0.1 \mathrm{mg} / \mathrm{mL},{ }^{30}$ and the solution was then sonicated using an ultrasonic probe ( $400 \mathrm{~W}, 10$ times). The suspension was then filtered through a membrane filter (Whatman, Maidstone, UK) with a pore size of $100 \mathrm{~nm}$ to remove the excess surfactants, rinsed thoroughly with ultrapure $\mathrm{H}_{2} \mathrm{O}$, and then centrifuged at $4000 \mathrm{rpm}$ for 15 minutes and repeated to remove macromolecular particles.

\section{Preparation of SWNT-NGR-DTX} NGR (CNGRCK $\mathrm{HK}_{3} \mathrm{HK}_{11}$ ) water solution was added to the SWNT-DTX suspension, which was modified by surfactant, and DPM with NGR and maleimide in a molar ratio of 1:20. ${ }^{31}$ After being left to stand overnight at room temperature, SWNT-NGR-DTX was obtained. The solid was washed with ultrapure $\mathrm{H}_{2} \mathrm{O}$ through a membrane filter to remove excess NGR.

Thin layer silica gel chromatography using n-butanolwater-acetic acid (4:2:1) as the developing agent and ninhydrin solution $0.5 \%$ as the coloring agent were used to test for conjugation of NGR to SWNT-DTX.

\section{Characterization of SWNT-NGR-DTX Determination of loading efficiency of docetaxel}

SWNT-NGR-DTX was diluted with acetonitrile and sonicated to ensure that the docetaxel was dissolved completely, then centrifuged to separate the SWNT and docetaxel to determine the amount of docetaxel bound to the SWNT. The concentration of docetaxel in acetonitrile was determined by high-pressure liquid chromatography. The chromatographic conditions were set as follows: an Eclipse XDB-C18 column $(150 \mathrm{~mm} \times 4.6 \mathrm{~mm}, 5.0 \mu \mathrm{m})$; mobile phase acetonitrile/ water 50:50; column temperature $30^{\circ} \mathrm{C}$; detection wavelength $231 \mathrm{~nm}$; flow rate $1.0 \mathrm{~mL} /$ minute; and injection volume $20 \mu \mathrm{L}$. Absorbance at $808 \mathrm{~nm}\left(\mathrm{~A}_{808}\right)$ was used to determine the SWNT concentration in the suspension by visible spectrophotometry. ${ }^{29}$

Determination of size distribution and zeta potential A $100 \mu \mathrm{L}$ sample of SWNT-NGR-DTX nanosuspension was diluted with ultrapure $\mathrm{H}_{2} \mathrm{O}$ to $2 \mathrm{~mL}$. The size distribution and zeta potential of the nanosuspension was determined at $25^{\circ} \mathrm{C}$ using a Nano ZS-90 (Malvern Instruments, Worcestershire, UK).

\section{Transmission electron microscopy}

The SWNT-NGR-DTX nanosuspension was dripped onto the copper line and scanned by transmission electron microscopy (Tecnai $\mathrm{G}^{2} 20$ ) at $100 \mathrm{kV}$.

\section{Preparation of SWNT-NGR-DTX-FITC and cell uptake}

Fluorescein isothiocyanate (FITC) $0.1 \mathrm{~mL}$ in dimethyl sulfoxide $1 \mathrm{mg} / \mathrm{mL}$ was added to $5 \mathrm{~mL}$ of SWNT-NGR-DTX, and the mixture was then sonicated and protected from light. Gel chromatography was used to remove the excess FITC by loading $1 \mathrm{~mL}$ of the solution onto a Sephadex G-25 column (Sigma). When the elution solvent $\left(\mathrm{H}_{2} \mathrm{O}\right)$ was flown through the column, the formation of two separate yellow bands was observed. The fractions were collected, and the absorbance of the various fractions was measured at $488 \mathrm{~nm}$ with a spectrophotometer. Fractions from the first elution peak were pooled because they were attributed to the higher molecular weight SWNT-NGR-DTX-FITC conjugate (also confirmed by fluorescence measurement). ${ }^{29}$

PC3 cells were cultured in RPMI medium 1640 supplemented with $10 \%$ fetal bovine serum and penicillin-streptomycin $1 \%$. The incubations of SWNT-NGR-DTX-FITC with PC3 cells were carried out on cover glasses in six-well plates, with the cells having been seeded for 24 hours before incubation. SWNT-NGR-DTX-FITC was added to each well at a final SWNT concentration of $2.5-5 \mu \mathrm{g} / \mathrm{mL}$. The incubations were carried out at $37^{\circ} \mathrm{C}$ in $5 \% \mathrm{CO}_{2}$ for $0.5,1$, and 2 hours, respectively. After incubation, the cover glasses were washed with phosphate-buffered solution followed by soaking for 15 minutes in $4 \%$ polymerisatum, then washed with deionized water. The cells were imaged by a fluorescence microscope (Zeiss LSM 510). 


\section{Cytotoxicity}

PC3 cells were plated in 96-well plates and treated with different concentrations of SWNT-NGR for 72 hours to investigate the cytotoxicity of the delivery system alone, and then SWNT-NGR-DTX, SWNT-DTX, or docetaxel for 24, 48 , and 72 hours. The weight ratio of SWNT to docetaxel was $1: 1.2$. Cell viability was measured using the sulforhodamine B assay.

\section{Pharmacokinetics and biodistribution studies}

The concentrations of docetaxel in plasma from the tumorfree healthy mice were measured after intravenous injection of SWNT- NGR-DTX, SWNT-DTX, or docetaxel. The dose of docetaxel used in this study was chosen to approximate the intraperitoneal dosage of docetaxel used in humans $\left(75 \mathrm{mg} / \mathrm{m}^{2}\right)$.

The mice were fasted for 12 hours before injection, but had access to water ad libitum. The experimental groups and the control group were given SWNT-NGR-DTX, SWNT-DTX, and docetaxel, respectively (docetaxel in each group at $25.125 \mathrm{mg} / \mathrm{kg}$ ). Blood samples were collected at $0.083,0.25,0.5,1,2$, and 4 hours after injection. At hours $0.5,1$, and 2 after administration, the tissues/organs were collected, weighed, and homogenized in buffer (acetonitrile to saline ratio,1:1) for biodistribution analyses. The weight ratio of tissues to buffer was 1:3. The tissues were then sonicated to a homogenate. Docetaxel concentrations in plasma were analyzed by high-pressure liquid chromatography.

\section{Animal model and treatment}

All care and treatment of the animals used in this study was performed in accordance with the guidelines for the Care and Use of Laboratory Animals published by the National Institutes of Health. The S180 tumor models were generated by subcutaneous injection of $2 \times 10^{6} \mathrm{~S} 180$ cells in $0.2 \mathrm{~mL}$ of phosphatebuffered solution into the right shoulder of female BALB/c mice. The mice were used for the study when the tumor volume reached $100 \mathrm{~mm}^{3}$ (7 days after tumor inoculation).

The animals were divided into five groups $(\mathrm{n}=6$ per group) to receive normal saline (controls), SWNT-NGR, docetaxel, SWNT-DTX, or SWNT-NGR-DTX. The injected doses were normalized to $25.125 \mathrm{mg} / \mathrm{kg}$ of docetaxel. The mice were observed daily for clinical symptoms, and the tumor sizes were measured by caliper on alternate days, and the volume calculated as (tumor width) $)^{2} \times($ tumor length) $/ 2$.

The study preparations were injected into the mice via the caudal tail vein every 2 days. After seven administrations, the mice were euthanized, and tissues from the heart, liver, spleen, lung, kidney, brain, thymus, and the tumors were harvested and soaked in 10\% formalin solution. The tissues collected were embedded in paraffin and mounted on slides stained with hematoxylin and eosin. Morphological changes were observed under a microscope.

\section{Laser radiation at $808 \mathrm{~nm}$}

For the ex vitro laser radiation experiments, SWNT-NGR-DTX solutions at high, middle, and low concentrations (SWNT approximately 8.3, 4.2 , and $0.9 \mu \mathrm{g} / \mathrm{mL}$; docetaxel about 10, 5 , and $1 \mu \mathrm{g} / \mathrm{mL}$ ) were irradiated by $808 \mathrm{~nm}$ laser at $1.4 \mathrm{~W} / \mathrm{cm}^{2}$, and the temperature was measured.

For the in vitro laser radiation experiments, PC3 were seeded in 96-well plates, and incubated with SWNT-NGRDTX at high, middle, and low concentrations, respectively. Six hours after treatment, the medium containing the drug was transferred to fresh medium, and the laser irradiation groups were put under an $808 \mathrm{~nm}$ laser for 1, 2, and 3 minutes. After 24 hours, a sulforhodamine B assay was performed to determine cell viability and calculate the inhibition rate.

The in vivo laser radiation experiment followed the same steps as for the animal model and treatment section described earlier, except that $808 \mathrm{~nm}$ laser irradiation was performed to tumor tissues in the S180 mice model for one minute after each administration.

\section{Statistical analysis}

Quantitative data were expressed as the mean \pm standard deviation. Means were compared using the Student's $t$-test with SPSS 17.0 statistical software (SPSS Inc, Chicago, IL), and $P$ values $<0.05$ were considered to be statistically significant.

\section{Results and discussion Preparation of soluble SWNT-DTX}

SWNT are not soluble in water, which greatly hinders their application as drug carriers. In this study, the dispersion effect of several surfactants on SWNT was examined. Lecithin High Potency, Poloxamer 188, HS 15, and PVPk30 were used at different concentrations. The loading rate was dosedependent using PVPk30, and reached a maximum (SWNT to docetaxel ratio about $1: 1.2$ ) at $10 \mathrm{mg} / \mathrm{mL}$ of PVPk30 (Figure 2).

Because SWNT is a new drug delivery carrier, there is not yet a well established method or procedure for its preparation. Our experiment adopts the method of saturated solution crystalization method, which means that docetaxel is dispersed 


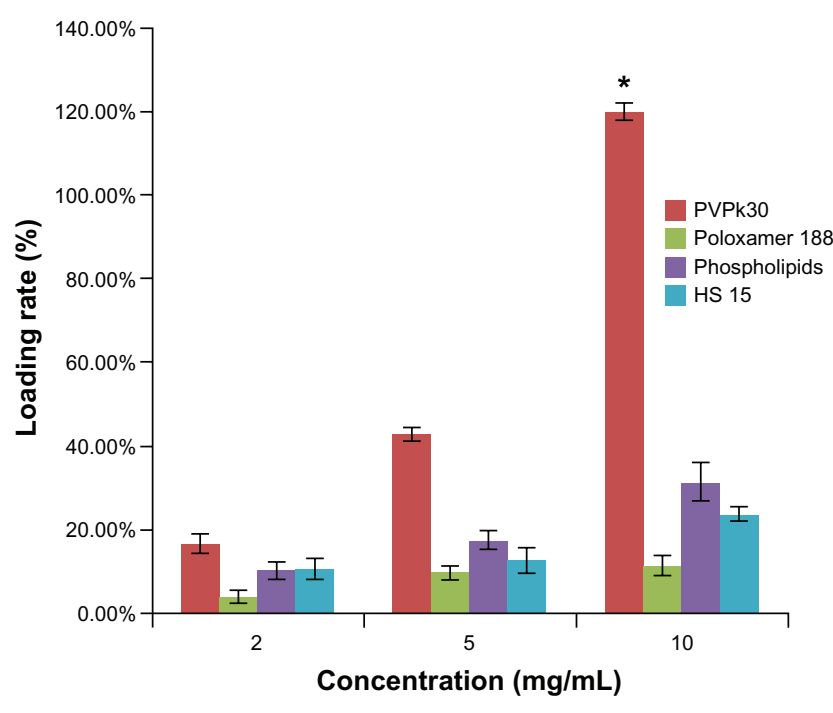

Figure 2 Effects of different surfactants on loading rate. Concentrations of PVPk30, Poloxamer 188, phospholipids, and HS 15 were 2,5 , and $10 \mathrm{mg} / \mathrm{mL}$, respectively. Notes: Data are presented as the mean \pm standard deviation $(n=3)$. $* P<0.0$ versus Poloxamer 188, phospholipids, and HS 15 at $10 \mathrm{mg} / \mathrm{mL}$, respectively.

into a molecular state using a soluble organic solvent, SWNT are added, and the water phase is then gradually added under ultrasound to reduce the solubility of docetaxel so that it can be separated out slowly and attached to SWNT with the influence of ultrasonic power. SWNT-DTX is precipitated by high-speed centrifugation, the organic solvent and water phase are removed, SWNT-DTX is dispersed again into aqueous solution with surfactants, and finally the suspension is centrifuged to remove the macromolecular particles.

During the preparation process, docetaxel was added to the SWNT, but they were difficult to adsorb due to their hydrophobic characteristics. Therefore, molecular docetaxel was added to make it fully exposed to the surface. When the phosphate-buffered water phase was dripped into the docetaxel-ethanol solution, docetaxel was gradually precipitated and adsorbed onto the SWNT and, using an ultrasonic probe instrument, more energy was applied to achieve stronger adsorption. Therefore, the power and frequency of the ultrasonic probe had a great impact on drug-loading efficiency.

Compared with zwitterionic surfactant, anionic surfactant has better dispersion and solubilization effects. However, due to its strong hemolytic effect, it cannot be used as an additive in injections. In this study, several surfactants for injection were chosen. Initially, SWNT were dispersed in water, and drugs were then added to the system, but only few were adsorbed to SWNT. The reason was probably that SWNT were wrapped in the surfactants, which prevented the drug from adsorption through $\pi-\pi$ stacking interactions.
We then adopted the nanoprecipitation method to absorb the drugs onto the surface of SWNT and then disperse the complex with surfactants, which significantly increased the drug loading rate.

\section{Preparation of SWNT-NGR-DTX}

With maleimide in the DSPE-PEG-maleimide conjugate, which could undergo an addition reaction with the double bond in sulfhydryl groups at room temperature, the compound reacted with cysteine in $\mathrm{CNGRCK}_{2} \mathrm{HK}_{3} \mathrm{HK}_{11}$ (C, containing sulfhydryl groups) and connected covalently. Thin layer silica gel chromatography results (Figure 3A) showed that there was no free NGR spot after reaction with NGR peptides, while there were visible NGR spots when they were mechanically mixed with SWNT, which demonstrated that NGR had completely attached to the surface of SWNT.

Size distribution is an important parameter for nanopreparation and hugely affects performance in vivo. As electric charges repel each other, potentials on the surface of the particles can prevent them from flocculation and keep the system stable. Charged particle dispersion is measured as the zeta potential, which is closely related to physical stability. The SWNT-NGR-DTX particle size was $182.8 \pm 2.8 \mathrm{~nm}$ (Figure 3D) and the zeta potential was $-12.06 \pm 0.71 \mathrm{mV}$ (Figure 3E). It is noteworthy that SWNT-NGR-DTX solution can keep a few weeks or even months without aggregation (Figure 3C).

SWNT showed a filamentous distribution, and docetaxel was attached to the surface of the SWNT (Figure 3B), which was reversible. Therefore, when the organism absorbed SWNT, docetaxel could be easily dissociated and take effect.

NGR plays the role of angiogenesis-targeting peptide in the preparation. If NGR is added before the surfactants, it will be adsorbed onto the surface of the SWNT and affect targeting effort, as SWNT also have the adsorbing effect towards protein. Therefore, SWNT-DTX is prepared first, then DSPE-PEG-maleimide is added into it. As in the phospholipids, the DSPE exerts a strong dispersing effect by adsorbing one end to SWNT, the long-chain PEG exposes maleimide, which induces an addition reaction with NGR, and NGR lies at the outer end of the complex, which assures its targeting effect.

\section{Cell uptake by SWNT-NGR-DTX-FITC}

The SWNT drug delivery system can effectively deliver drugs into cells and inhibit phagocytic ability. In this study, uptake of FITC, SWNT-DTX-FITC, and SWNT-NGRDTX-FITC by PC 3 cells was examined. With the properties 

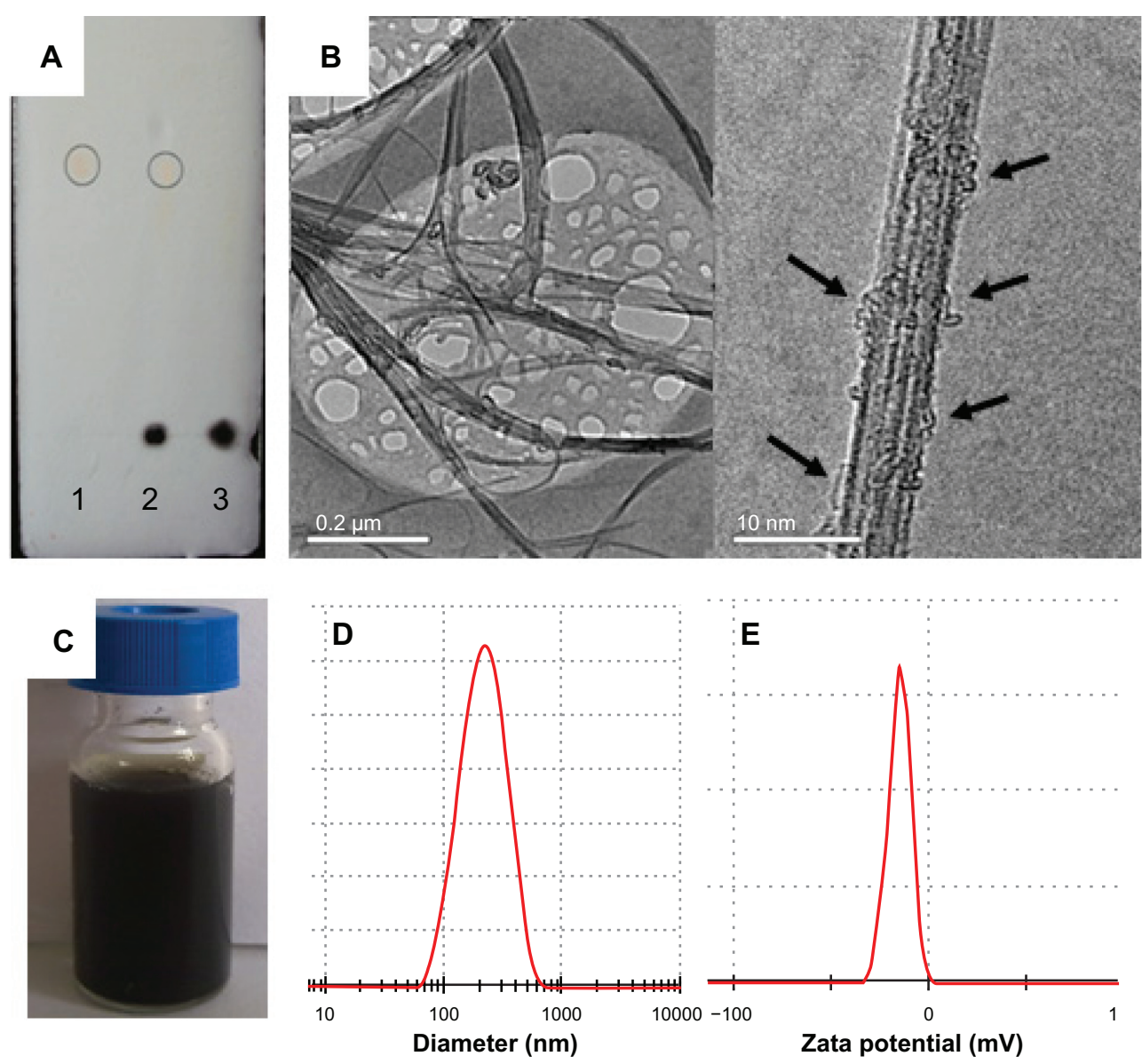

Figure 3 Characterization of SWNT-NGR-DTX. (A) Thin layer silica gel chromatography image of I-(NGR), 2-(NGR mechanical mixing with the SWNT-DTX), and 3-(SWNT-NGR-DTX); (B) transmission electron microscopic image of SWNT-NGR-DTX; (C) solubility of SWNT-NGR-DTX; (D) particle size of SWNT-NGR-DTX; and (E) zeta potential of SWNT-NGR-DTX.

Abbreviations: SWNT, single-walled carbon nanotubes; NGR, (Asn-Gly-Arg) peptide; DTX, docetaxel.

of the SWNT surface, it is easy to adsorb the large $\pi$ bond and cause a $\pi-\pi$ stacking effect. With multibenzenes in the FITC molecular structure, it can easily be adsorbed onto the surface of SWNT.

Fluorescence microscopy was applied to track the location of docetaxel and the fluorescein-labeled SWNT inside cells (Figure 4). As mentioned earlier, the drug delivery experiment is based on the hypothesis that functionalized SWNT are taken up by PC 3 cells through endocytosis. However, the FITC is mainly observed outside the cell. Although drugs can enter cells themselves, nanotubes still play an important role because they enable molecular targeting via the attachment of NGR peptides and are crucial for the delivery of drugs that are not taken up by cells under normal conditions. This experiment showed that uptake of SWNT-NGR-DTX-FITC and SWNT-DTX-FITC by PC3 cells was time-dependent, with uptake of all SWNT-NGR-DTX-FITC into PC3 cells at 2 hours, but only a part of SWNT-DTX-FITC was transferred into the cells at this time point (Figure 4). Apart from this, our results provide an answer to whether carbon nanotubes enter the nuclei of cells. This study clearly shows the nanotubes located in the cytoplasm under the applied conditions.

\section{Cell viability}

The concentration of docetaxel was $8 \mu \mathrm{g} / \mathrm{mL}$ and for SWNT was about $6.7 \mu \mathrm{g} / \mathrm{mL}$. When the concentration of SWNT was below $6.7 \mu \mathrm{g} / \mathrm{mL}$, the SWNT-NGR had no signifcant cytotoxicity to the PC3 cells (Figure 5), but proliferation of the PC3 cells was inhibited in the docetaxel, SWNT-DTX, and SWNT-NGR-DTX groups, with the inhibition rate being positively correlated with dose and time. The corresponding inhibition rates at each time point for the docetaxel and SWNT-NGR-DTX groups were significantly different (Figure 6). However, at the time points of 48 hours and 72 hours, as shown in Figure 6, there is no significant difference between SWNT-NGR-DTX, SWNT-DTX, and docetaxel. This is probably due to the fact that the targeted delivery system can be transferred faster into cells, and as a result, its effect 


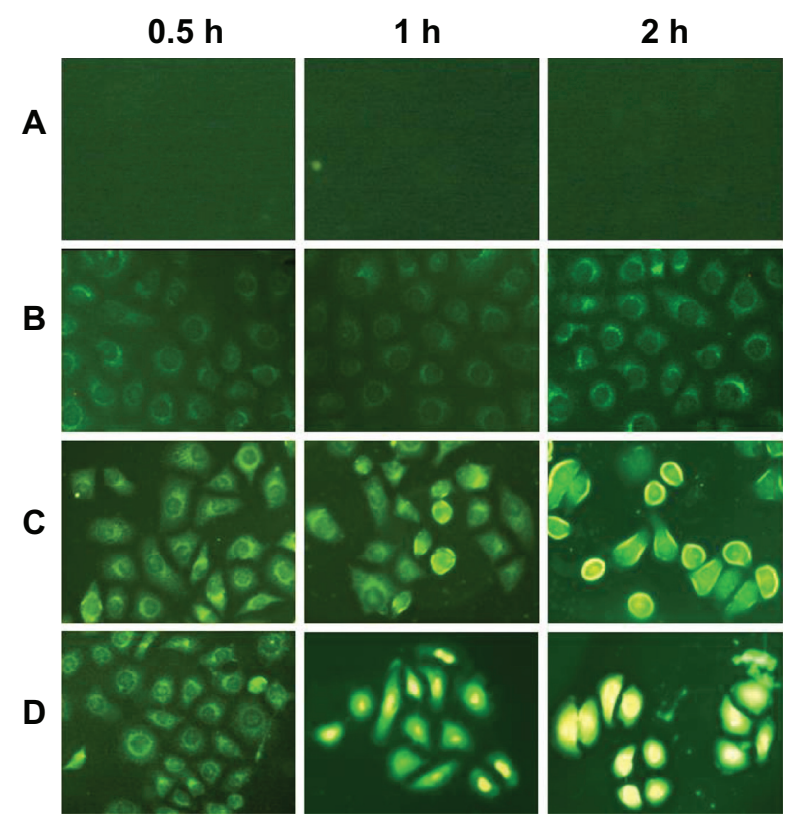

Figure 4 Fluorescence microscopic images of PC3 cells. (A) control group; (B) FITC alone; (C) SWNT-DTX-FITC; and (D) SWNT-NGR-DTX-FITC at 0.5, I, and 2 hours.

Abbreviations: SWNT, single-walled carbon nanotubes; NGR, (Asn-Gly-Arg) peptide; DTX, docetaxel; FITC, fluorescein isothiocyanate.

is significantly better than for the other two groups in the early stages. However, when the time periods are prolonged to 48 hours and 72 hours, the docetaxel in all groups is transferred into the cells, so the advantage is weakened.

\section{Pharmacokinetics and biodistribution}

The pharmacokinetics of docetaxel, SWNT-DTX, and SWNT-NGR-DTX were investigated in the blood samples by high-pressure liquid chromatography (Figure 7), showing that the rate of decrease of docetaxel was faster than for

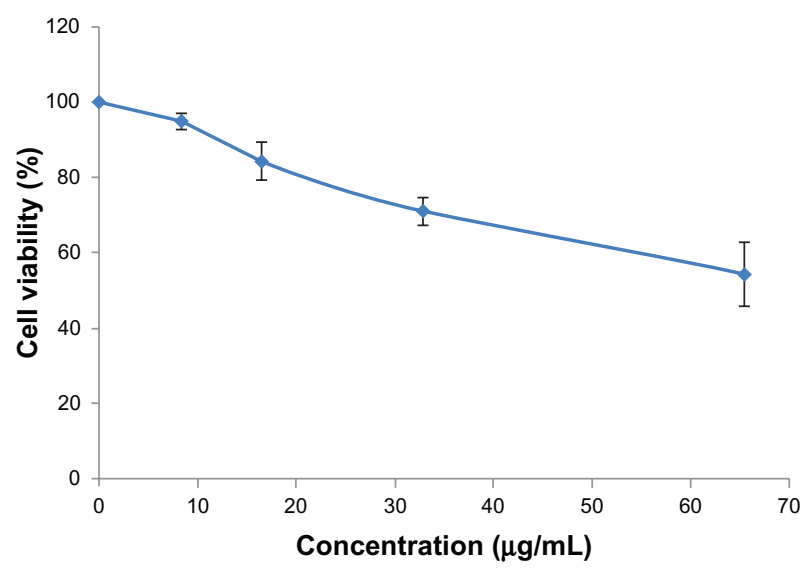

Figure 5 Cytotoxicity of SWNT-NGR in PC3 cells.

Note: Data are presented as the mean \pm standard deviation $(n=3)$.

Abbreviations: SWNT, single-walled carbon nanotubes; NGR, (Asn-Gly-Arg) peptide.
SWNT-DTX and SWNT-NGR-DTX after administration. The blood concentration of docetaxel rapidly declined to the quantitation limit, while the decreasing rate of SWNT-NGRDTX and SWNT-DTX was evidently slower, the decreasing rates in the SWNT-DTX and SWNT-NGR-DTX groups were not significantly different, but the rates of both were markedly different from that of the docetaxel group.

The area under the curve for SWNT-NGR-DTX was about six and 64 times greater than for SWNT-DTX and docetaxel, respectively, while the elimination rate was less (Table 1) and mean residence time was 1.6 and 2.6 times longer, respectively (Table 2). This result is attributed to the high hydrophobicity of conjugated docetaxel, reducing the biological inertness of docetaxel in vivo and shortening the blood circulation time. This shows that SWNT-NGRDTX significantly increased the blood circulation time of docetaxel.

To clarify the tumor treatment efficacy of the various docetaxel formulations (SWNT-NGR-DTX, SWNT-DTX, and docetaxel), the biodistribution of docetaxel was investigated in tumors and various organs in the mice. For quantitative evaluation of the targeting characteristics of SWNT-NGRDTX in vivo, several parameters were applied, including overall targeting efficiency (TE), ie, the percentage of drug distribution in the tissues. The formula, applicable to all tissues, is as follows:

$$
T E=\frac{\left(A U C_{0 \rightarrow 3 h}\right) i}{\sum_{i=1}^{n}\left(A U C_{0 \rightarrow 3 h}\right)} \times 100 \%
$$

The calculation of $A U C_{0 \rightarrow 3 h}$ was performed using a trapezoidal method. Significant differences were observed for the biodistribution of docetaxel administered in the three different formulations (Figure $8 \mathrm{~B}$ ).

The $A U C_{0 \rightarrow 3 h}$ of docetaxel in SWNT-NGR-DTX for the liver, spleen, lung, kidney, and tumor tissue was markedly larger than in the docetaxel control group. Compared with the SWNT-DTX group, distribution of SWNT-NGR-DTX in the liver, spleen, lung, and kidney was reduced, while distribution in tumor tissues was significantly increased, and little docetaxel reached the tumor tissues when the drug was administered alone (Figure 8B). It was demonstrated that SWNT functionalized by the PVPk30 method has a particular distribution pattern, so eventually the targeting efficiency of SWNT-NGR-DTX was similar or even less in tumor tissue than in other organs. However, the NGR effect can reduce the distribution of SWNT-DTX in other organs, 

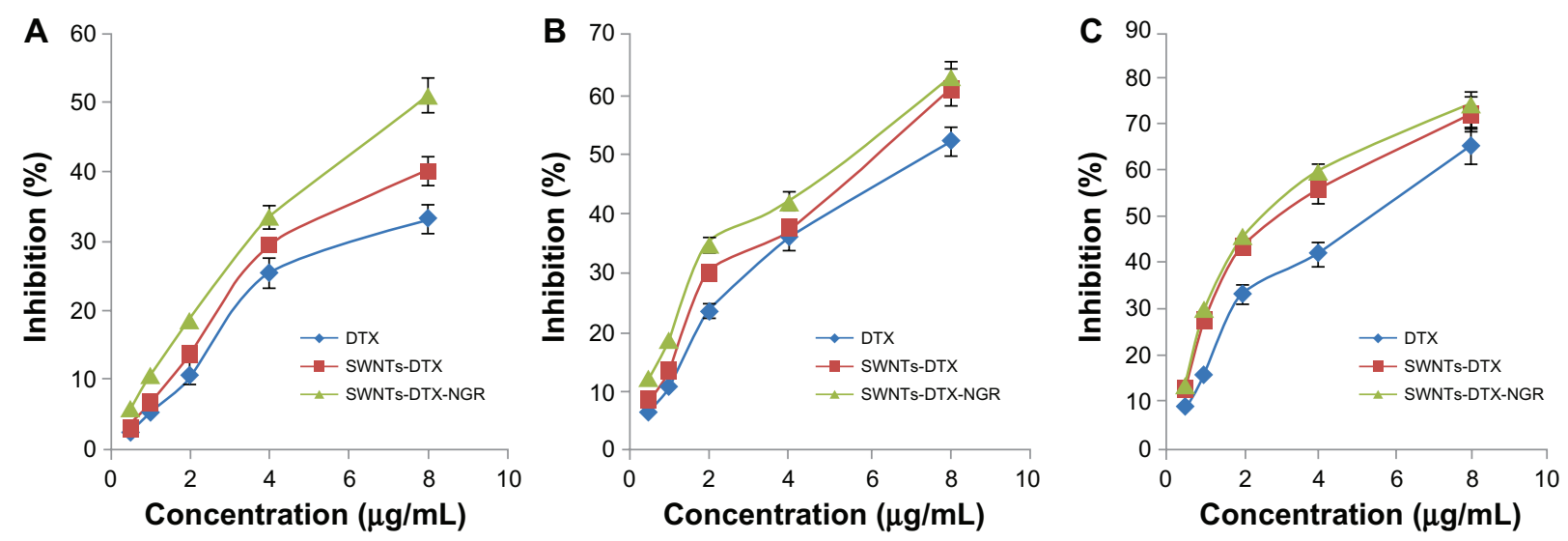

Figure 6 Inhibition rate of docetaxel, SWNT-DTX, and SWNT-NGR-DTX in PC3 cells. SWNT-NGR-DTX was significantly different $(P<0.05)$ from the other groups at 24 hours (A) but at 48 hours $(\mathbf{B})$ and 72 hours $(\mathbf{C})$ there was no significant difference between SWNT-DTX and SWNT-NGR-DTX, while the SWNT-NGR-DTX group was significantly different from the docetaxel group $(P<0.05)$.

Note: Data are presented as the mean \pm standard deviation $(n=3)$.

Abbreviations: SWNT, single-walled carbon nanotubes; NGR, (Asn-Gly-Arg) peptide; DTX, docetaxel.

and increase distribution in the tumor to achieve better treatment of cancer.

The targeting efficiency values for SWNT-NGR-DTX in the liver and tumor were higher than in the docetaxel control group (Figure 8A), indicating good targeting of SWNT-NGR-DTX to the liver and tumor tissues. The concentration of docetaxel in each tissue was increased by SWNT-DTX, which should enhance the therapeutic effect to a certain extent.

\section{Laser radiation}

In this study, three different concentrations of SWNTNGR-DTX were irradiated under laser, and temperature changes with time were measured. The heating efficiency of

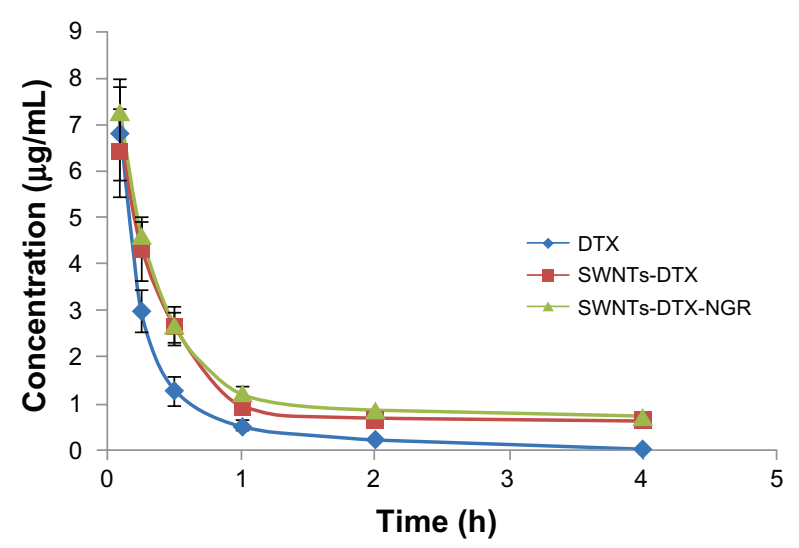

Figure 7 Mean docetaxel concentration in plasma after intravenous administration of docetaxel, SWNT-DTX, and SWNT-NGR-DTX (mean \pm standard deviation, $n=5$ ). The decreasing rates of SWNT-DTX and SWNT-NGR-DTX were not significantly different, but the rate of both were markedly different from that in the docetaxel group $(P<0.05)$.

Abbreviations: SWNT, single-walled carbon nanotubes; NGR, (Asn-Gly-Arg) peptide; DTX, docetaxel.
SWNT-NGR-DTX relies strongly on time and dose, meaning that with increasing concentration and time, the temperature was significantly higher (Figure 9). Under laser irradiation at $808 \mathrm{~nm}$, SWNT-NGR-DTX showed strong light-heat transfer characteristics.

The inhibition rate of the SWNT-NGR-DTX complex towards PC3 cells was significantly increased under $808 \mathrm{~nm}$ near-infrared laser radiation (Figure 10). When the concentration of SWNT was $14.4 \mu \mathrm{g} / \mathrm{mL}$, the rate of inhibition reached $95.33 \%$ after radiation for one minute. When the SWNT-NGR-DTX complex containing SWNT $7.3 \mu \mathrm{g} / \mathrm{mL}$ was irradiated for one minute, the inhibition rate was about $35 \%$, but reduced to $90.73 \%$ if irradiated for two minutes. This is probably due to the fact that cell tolerance drops dramatically at a certain temperature during heat treatment, as it does for SWNT of different concentrations over different

Table I The pharmacokinetic parameters of DTX after intravenous administration of DTX, SWNT-DTX, and SWNTNGR-DTX in mice

\begin{tabular}{lllll}
\hline Parameter & Unit & SWNT-DTX & $\begin{array}{l}\text { SWNT-NGR- } \\
\text { DTX }\end{array}$ & DTX \\
\hline $\mathrm{V}(\mathrm{c})$ & $\mathrm{L} / \mathrm{kg}$ & 0.643 & 0.547 & 0.477 \\
$\mathrm{t}_{1 / 2} \alpha$ & $\mathrm{h}$ & 0.264 & 0.226 & 0.123 \\
$\mathrm{t}_{1 / 2} \beta$ & $\mathrm{h}$ & 148.948 & 738.729 & 12.728 \\
$\mathrm{~K}_{21}$ & $\mathrm{~L} / \mathrm{h}$ & 0.198 & 0.245 & 0.357 \\
$\mathrm{~K}_{10}$ & $\mathrm{~L} / \mathrm{h}$ & 0.062 & 0.012 & 0.860 \\
$\mathrm{~K}_{12}$ & $\mathrm{~L} / \mathrm{h}$ & 2.367 & 2.813 & 4.479 \\
$\mathrm{AUC}$ & $(\mu \mathrm{g} / \mathrm{mL}) \cdot \mathrm{h}$ & 125.952 & 777.614 & 12.188 \\
$\mathrm{CL}(\mathrm{s})$ & $\mathrm{mL} / \mathrm{h} / \mu \mathrm{g}$ & 0.0396 & 0.00643 & 0.410 \\
\hline
\end{tabular}

Note: All data are the average of five measurements.

Abbreviations: AUC, area under the curve; $\mathrm{CL}$, clearance; $\mathrm{V}$, volume of distribution; $t_{1 / 2}$, elimination half-life; $K$, elimination rate constant; SWNT, single-walled carbon nanotubes; NGR, (Asn-Gly-Arg) peptide; DTX, docetaxel; NS, normal saline. 
Table 2 Pharmacokinetic parameters calculated by statistical moment for mice treated with intravenous DTX, SWNT-DTX, and SWNT-NGR-DTX

\begin{tabular}{lllll}
\hline Parameters & Unit & $\begin{array}{l}\text { SWNT- } \\
\text { DTX }\end{array}$ & $\begin{array}{l}\text { SWNT-NGR- } \\
\text { DTX }\end{array}$ & DTX \\
\hline AUC $(\mathrm{S} 0)$ & $(\mu \mathrm{g} / \mathrm{mL}) \cdot \mathrm{h}$ & 9.888 & 14.628 & 7.470 \\
AUMC $(\mathrm{SI})$ & $(\mu \mathrm{g} / \mathrm{mL}) \cdot \mathrm{h}$ & 58.802 & 144.793 & 28.274 \\
AUMC $(\mathrm{S} 2)$ & $(\mu \mathrm{g} / \mathrm{mL}) \cdot \mathrm{h}$ & 886.286 & 3426.139 & 187.986 \\
MRT & $\mathrm{h}$ & 5.947 & 9.898 & 3.785 \\
VRT & $\mathrm{h} \cdot \mathrm{h}$ & 54.268 & 136.238 & 10.893 \\
\hline
\end{tabular}

Abbreviations: AUC, area under the curve; AUMC, area under the first moment of the plasma concentration-time curve; MRT: mean retention time; VRT, variance of residence time; SWNT, single-walled carbon nanotubes; NGR, (Asn-Gly-Arg) peptide; DTX, docetaxel.

time periods, resulting in an increased inhibition rate for SWNT-NGR-DTX from one minute to 2 minutes. The results show that SWNT has a significant photothermal effect, and when combined with chemotherapy, ideal antitumor effects could be reached.

Changes in tumor volume in the S180 mouse model are shown in Figure 11A. Compared with the normal saline group, the tumor volume in the docetaxel, SWNT-DTX, and SWNT-NGR-DTX groups showed a decrease one week after administration, but there were some remarkable differences. Efficacy in the SWNT-NGR-DTX and SWNT-DTX groups was superior to that in the docetaxel group, and both were markedly different from the docetaxel group. However, no significant variation was observed between the SWNT and normal saline groups.

In vivo, the results of the laser irradiation experiment (Figure 11B) showed that tumor volumes in the normal saline-laser and docetaxel-laser groups were both decreased when compared with the normal saline and docetaxel groups, respectively. There was a significant difference, indicating that radiation had an antitumor effect. The statistical results indicated a marked difference and better outcome in the SWNT-NGR-laser group than in the normal saline, normal saline-laser, and SWNT-NGR groups. The SWNT-NGR-DTX-laser group showed the best antitumor results and a strong inhibitory effect, even in the early stage of treatment. The results confirmed that radiation at $808 \mathrm{~nm}$ can significantly enhance the tumor inhibition effect.

In recent years, photothermal therapy based on nanomaterials has been suggested as a highly efficient therapeutic technique. Moon et al used PL-PEG2000- $\mathrm{NH}_{2}$ to functionalize SWNT, and demonstrated that the photothermal effect of PEG-SWNT exposed to near-infrared irradiation can destroy solid malignant tumors in vivo. SWNT-based photothermal
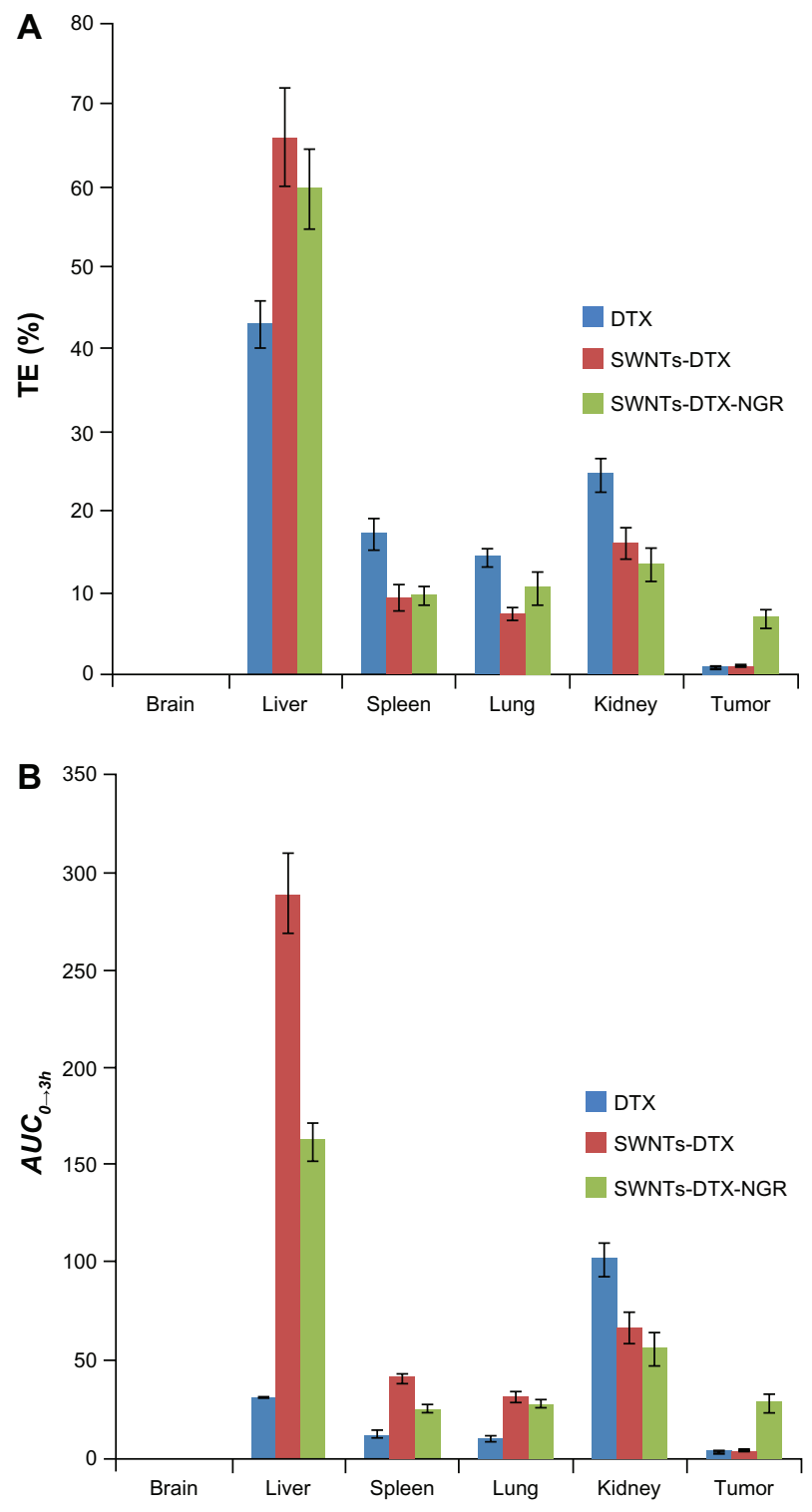

Figure 8 Targeting efficiency (A) and $A \cup C_{0 \rightarrow 3 h}$ of DTX concentration (B) in tissues of mice after intravenous administration of DTX, SWNT-DTX, and SWNT-NGR-DTX. Note: Data are presented as the mean \pm standard deviation, $(n=6)$.

Abbreviations: SWNT, single-walled carbon nanotubes; NGR, (Asn-Gly-Arg) peptide; DTX, docetaxel; AUC, area under the concentration versus time curve.

treatment achieved complete eradication of solid malignant tumors without toxicity or recurrence of tumors for a long period of time. ${ }^{27}$ Using PEG-PMHC18-coated SWNT, Liu et al carried out a pilot in vivo photothermal therapy study and observed promising efficacy in cancer cells. Their results highlight the importance of surface coating for the in vivo behavior of nanomaterials in general, and could provide guidelines for the future design of SWNT bioconjugates for various in vivo applications. ${ }^{25}$ Yang et al used nanographene sheets with polyethylene glycol (PEG) coating to exploit the strong optical absorbance of nanographene sheets in the near-infrared region for in vivo photothermal therapy, 


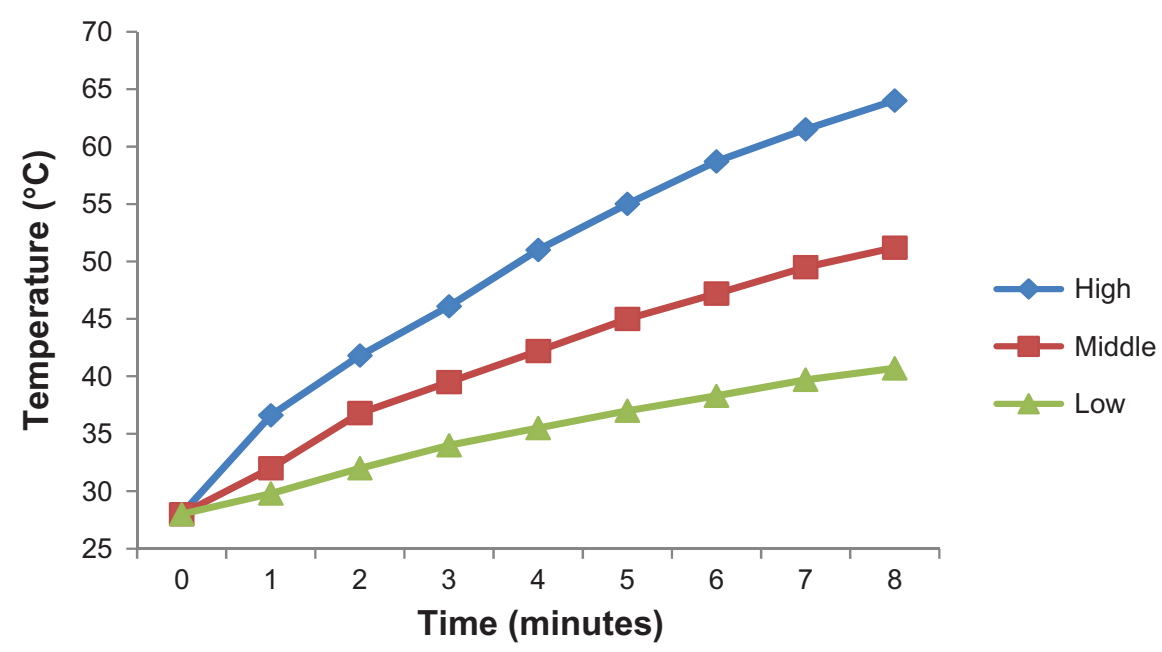

Figure 9 Temperature evolution of high, middle, and low concentrations of SWNT-NGR-DTX (SWNT approximately 8.3, 4.2, and 0.9 $\mu$ g/mL; DTX approximately I0, 5, and I $\mu \mathrm{g} / \mathrm{mL}$ ), respectively, during continuous radiation by $808 \mathrm{~nm}$ laser at $1.4 \mathrm{~W} / \mathrm{cm}^{2}$.

Abbreviations: SWNT, single-walled carbon nanotubes; NGR, (Asn-Gly-Arg) peptide; DTX, docetaxel.

achieving ultraefficient tumor ablation after intravenous administration of nanographene sheets and low-power nearinfrared laser irradiation to the tumor. ${ }^{32}$ SWNT functionalized by PEGylated phospholipids are biologically nontoxic and long-circulating nanomaterials with intrinsic near-infrared photoluminescence..$^{28}$ All the studies shared the same feature of modifying SWNT with PEG. Our study adopts the method of surfactant modification, whereby DPM is used at a low dose to conjugate with NGR, and the effect of using heat with this method is still significant.

\section{Histological analysis}

To investigate the influence of SWNT-NGR-DTX on the organs of mice, a histological assay was conducted with paraffin-embedded sections and hematoxylin and eosin

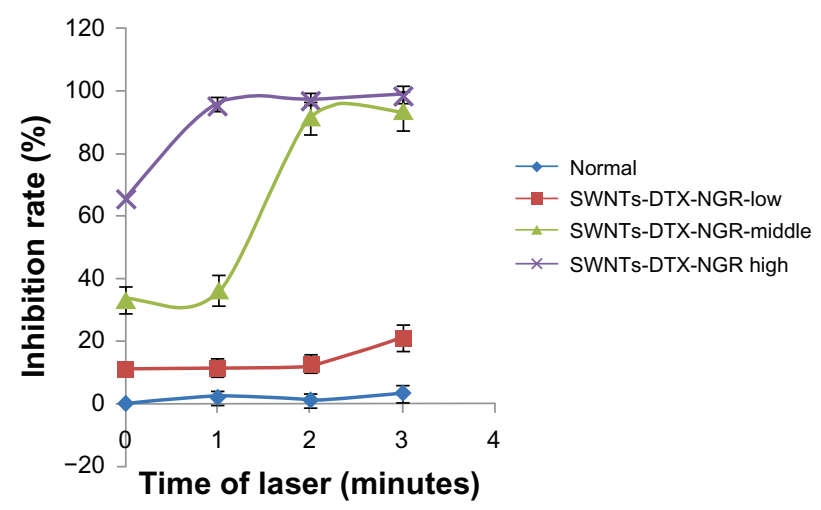

Figure 10 Inhibition by SWNT-NGR-DTX of PC3 cells under $808 \mathrm{~nm}$ laser irradiation using different concentrations and different laser times. Data presented as the mean \pm standard deviation $(n=3)$.

Abbreviations: SWNT, single-walled carbon nanotubes; NGR, (Asn-Gly-Arg) peptide; DTX, docetaxel. staining (Figure 12) which showed that, compared with the control group, there were no significant differences between the various tissues. However, tumor cells in the control group showed vigorous growth, a tight arrangement, a large body and intact shape, while cell necrosis, lysis, and fragmentation
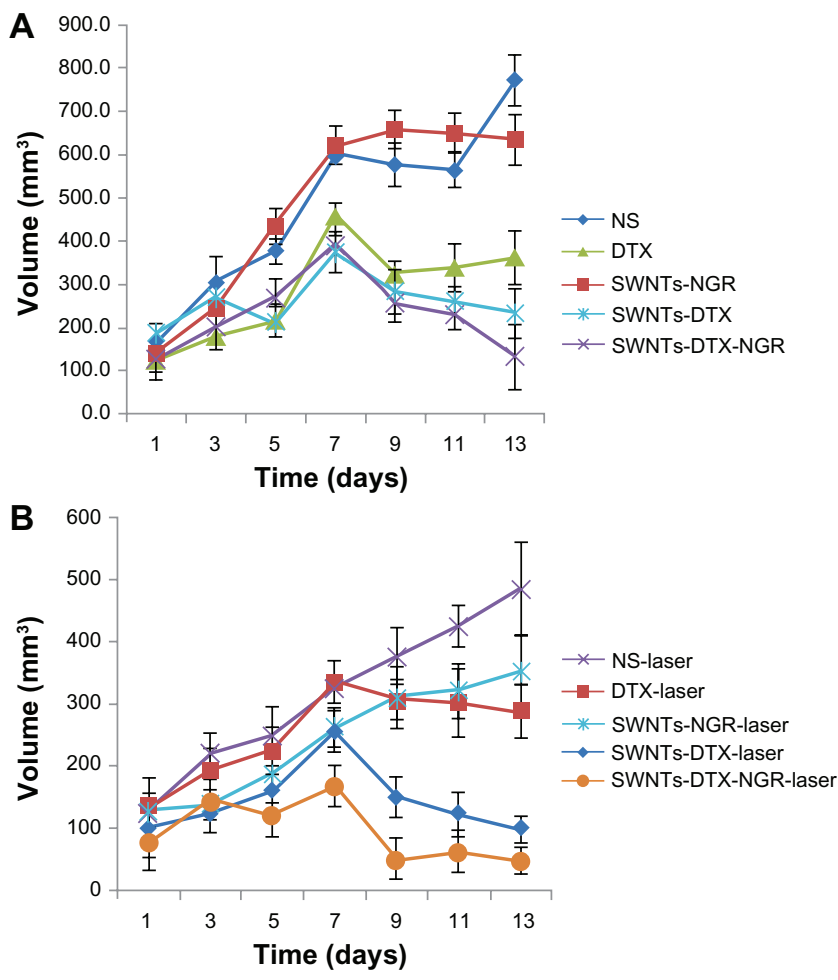

Figure II Average tumor volume in a SI80 mouse model of treatment without (A) and with (B) laser in vivo. The SWNT-NGR-DTX-laser group shows significant $(P<0.05)$ suppression of tumor growth compared with the other experimental groups $(\mathrm{n}=6)$.

Abbreviations: SWNT, single-walled carbon nanotubes; NGR, (Asn-Gly-Arg) peptide; DTX, docetaxel; NS, normal saline. 


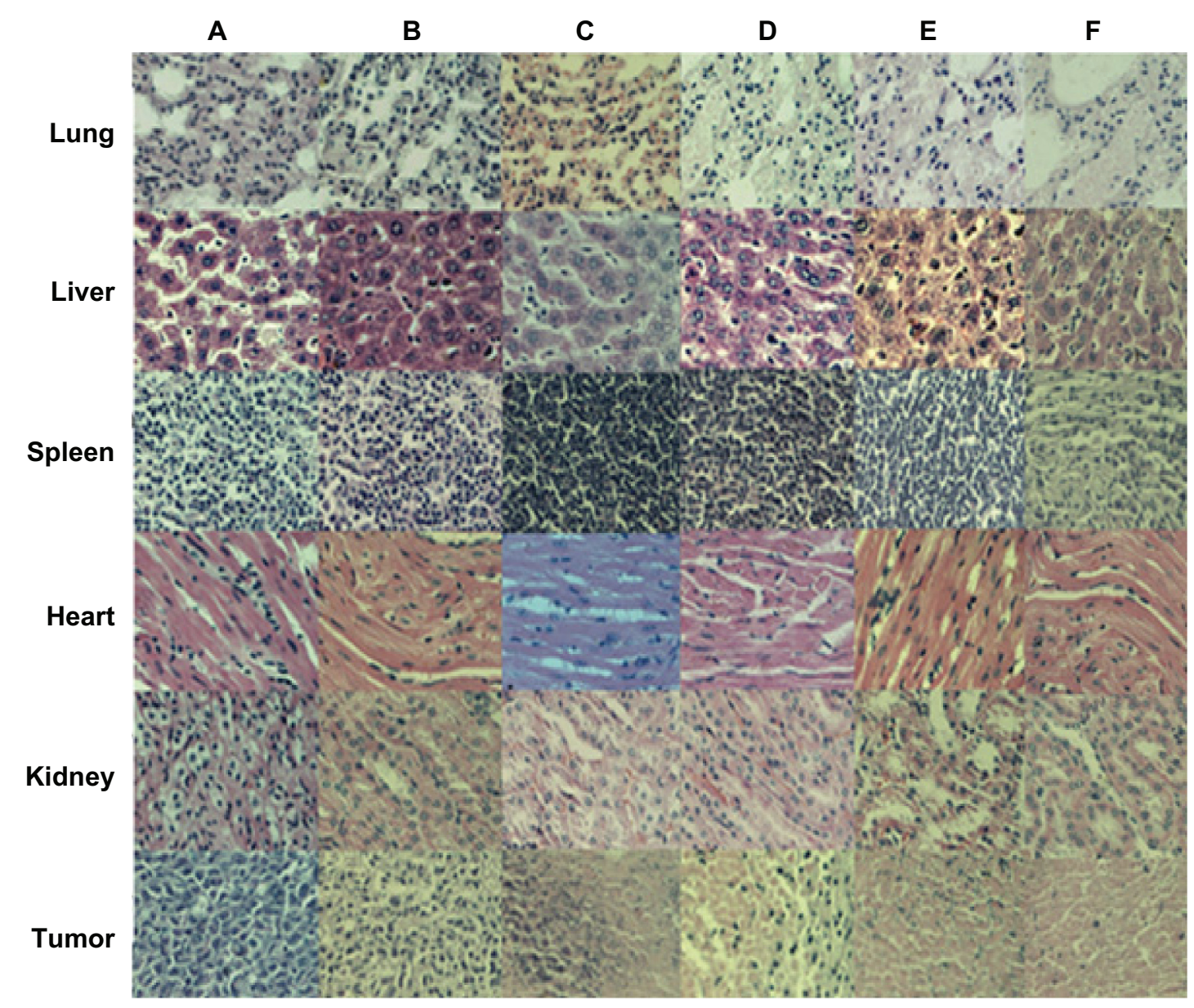

Figure 12 Histologic assessments of major organs and tumor tissues with hematoxylin and eosin staining in mice (200x). (A) NS, (B) SWNT, (C) SWNT-DTX, (D) DTX, (E) SWNT-NGR-DTX, and (F) SWNT-NGR-DTX-laser.

Abbreviations: SWNT, single-walled carbon nanotubes; NGR, (Asn-Gly-Arg) peptide; DTX, docetaxel; NS, normal saline.

occurred to a certain extent in the docetaxel, SWNT-DTX, SWNT-NGR-DTX, and SWNT-NGR-DTX-laser groups. No significant morphological difference was found between the SWNT group and the control group.

\section{Conclusion}

In summary, a new anticancer drug delivery system using docetaxel as a model, ie, SWNT-NGR-DTX, was developed to identify the biodistribution characteristics of SWNT and their effect on cancer cells when mediated by thermal therapy. The targeting efficiency of SWNT-NGR-DTX in tumor tissue was enhanced compared with SWNT-DTX and docetaxel. It is suggested that this SWNT drug delivery system may be promising in terms of treatment efficacy for cancer and with minimal side effects.

\section{Disclosure}

The authors declare no conflicts of interest in relation to this work.

\section{References}

1. Krajcik R, Jung A, Hirsch A, Neuhuber W, Zolk O. Functionalization of carbon nanotubes enables non-covalent binding and intracellular delivery of small interfering RNA for efficient knock-down of genes. Biochem Biophys Res Commun. 2008;369(2):595-602.

2. Ji SR, Liu C, Zhang B, et al. Carbon nanotubes in cancer diagnosis and therapy. Biochim Biophys Acta. 2010;1806(1):29-35.

3. Mahmood M, Karmakar A, Fejleh A, et al. Synergistic enhancement of cancer therapy using a combination of carbon nanotubes and anti-tumor drug. Nanomedicine (Lond). 2009;4(8):883-893.

4. Mielcarek J, Skupin P. Functionalization of carbon nanotubes for multimodal drug delivery. Przegl Lek. 2011;68(3):167-170. Polish.

5. Chen J, Shuji C, Zhao X, et al. Functionalized single-walled carbon nanotubes as rationally designed vehicles for tumor-targeted drug delivery. J Am Chem Soc. 2008;130(49):16778-16785.

6. Khazaei A, Rad MN, Borazjani MK. Organic functionalization of single-walled carbon nanotubes (SWCNTs) with some chemotherapeutic agents as a potential method for drug delivery. Int J Nanomedicine. 2010;5:639-645.

7. Chen Q, Wang Q, Liu YC, et al. Energetics investigation on encapsulation of protein/peptide drugs in carbon nanotubes. $J$ Chem Phys. 2009;131(1):015101.

8. Cheung W, Pontoriero F, Taratula O, Chen AM, He H. DNA and carbon nanotubes as medicine. Adv Drug Deliv Rev. 2010;62(6):633-649.

9. Varkouhi AK, Foillard S, Lammers T, et al. SiRNA delivery with functionalized carbon nanotubes. Int J Pharm. 2011;416(2):419-425. 
10. Ciofani G, Raffa V, Vittorio $\mathrm{O}$, et al. In vitro and in vivo biocompatibility testing of functionalized carbon nanotubes. Methods Mol Biol. 2010; 625:67-83.

11. Liu Z, Chen K, Davis C, et al. Drug delivery with carbon nanotubes for in vivo cancer treatment. Cancer Res. 2008;68(16):6652-6660.

12. Zhang Y, Bai Y, Yan B. Functionalized carbon nanotubes for potential medicinal applications. Drug Discov Today. 2010;15(11-12):428-435.

13. Pasqualini R, Koivunen E, Kain R, et al. Aminopeptidase $\mathrm{N}$ is a receptor for tumor-homing peptides and a target for inhibiting angiogenesis. Cancer Res. 2000;60(3):722-727.

14. Oostendorp M, Douma K, Hackeng TM, van Zandvoort MA, Post MJ, Backes WH. Pharmacokinetics of contrast agents targeted to the tumor vasculature in molecular magnetic resonance imaging. Contrast Media Mol Imaging. 2010;5(1):9-17.

15. Zhang Z, Wang B, Li H, Zhou T, Zhang L. The inhibitory effects of nanosize delivery system for antisense oligonucleotide of hTERT on EC9706 cells. Cancer Biol Ther. 2007;6(3):329-334.

16. Li H, Wang J, Zhou T, Zhang Y, Zhang Z. An investigation of the effects of nanosize delivery system for antisense oligonucleotide on esophageal squamous cancer cells. Cancer Biol Ther. 2008;7(11):1852-1859.

17. Zhao BJ, Ke XY, Huang Y, et al. The antiangiogenic efficacy of NGRmodified PEG-DSPE micelles containing paclitaxel (NGR-M-PTX) for the treatment of glioma in rats. J Drug Target. 2011;19(5):382-390.

18. Wang X, Wang Y, Chen X, Wang J, Zhang X, Zhang Q. NGR-modified micelles enhance their interaction with CD13-overexpressing tumor and endothelial cells. J Control Release. 2009;139(1):56-62.

19. [No authors listed]. Docetaxel and adjuvant treatment of breast cancer. Prescrire Int. 2011;20(117):149.

20. De Souza R, Zahedi P, Moriyama EH, Allen CJ, Wilson BC, PiquetteMiller M. Continuous docetaxel chemotherapy improves therapeutic efficacy in murine models of ovarian cancer. Mol Cancer Ther. 2010; 9(6):1820-1830.

21. Saloustros E, Georgoulias V. Docetaxel in the treatment of advanced non-small-cell lung cancer. Expert Rev Anticancer Ther. 2008;8(8): $1207-1222$.
22. Caponigro F, Longo F, Perri F, Ionna F. Docetaxel in the management of head and neck cancer. Anticancer Drugs. 2009;20(8):639-645.

23. Gueritte-Voegelein F, Guenard D, Lavelle F, Le Goff MT, Mangatal L, Potier P. Relationships between the structure of taxol analogues and their antimitotic activity. J Med Chem. 1991;34(3):992-998.

24. Huang N, Wang H, Zhao J, Lui H, Korbelik M, Zeng H. Single-wall carbon nanotubes assisted photothermal cancer therapy: animal study with a murine model of squamous cell carcinoma. Lasers Surg Med. 2010;42(9):638-648.

25. Liu X, Tao H, Yang K, Zhang S, Lee ST, Liu Z. Optimization of surface chemistry on single-walled carbon nanotubes for in vivo photothermal ablation of tumors. Biomaterials. 2011;32(1):144-151.

26. Zheng X, Zhou F. Noncovalent functionalization of single-walled carbon nanotubes by indocyanine green: Potential nanocomplexes for photothermal therapy. J Xray Sci Technol. 2011;19(2):275-284.

27. Moon HK, Lee SH, Choi HC. In vivo near-infrared mediated tumor destruction by photothermal effect of carbon nanotubes. ACS Nano. 2009;3(11):3707-3713.

28. Robinson JT, Welsher K, Tabakman SM, et al. High performance in vivo near-IR $(>1 \mathrm{~mm})$ imaging and photothermal cancer therapy with carbon nanotubes. Nano Res. 2010;3(11):779-793.

29. Kam NW, O'Connell M, Wisdom JA, Dai H. Carbon nanotubes as multifunctional biological transporters and near-infrared agents for selective cancer cell destruction. Proc Natl Acad Sci U S A. 2005;102(33): $11600-11605$.

30. Zhang Y, Jeong Lee H, Boado RJ, Pardridge WM. Receptor-mediated delivery of an antisense gene to human brain cancer cells. J Gene Med. 2002;4(2):183-194.

31. Lu J, Jeon E, Lee BS, Onyuksel H, Wang ZJ. Targeted drug delivery crossing cytoplasmic membranes of intended cells via ligand-grafted sterically stabilized liposomes. J Control Release. 2006;110(3): 505-513.

32. Yang K, Zhang S, Zhang G, Sun X, Lee ST, Liu Z. Graphene in mice: ultrahigh in vivo tumor uptake and efficient photothermal therapy. Nano Lett. 2010;10(9):3318-3323.
International Journal of Nanomedicine

\section{Publish your work in this journal}

The International Journal of Nanomedicine is an international, peerreviewed journal focusing on the application of nanotechnology in diagnostics, therapeutics, and drug delivery systems throughout the biomedical field. This journal is indexed on PubMed Central, MedLine, CAS, SciSearch $\AA$, Current Contents $\AA /$ Clinical Medicine,

\section{Dovepress}

Journal Citation Reports/Science Edition, EMBase, Scopus and the Elsevier Bibliographic databases. The manuscript management system is completely online and includes a very quick and fair peer-review system, which is all easy to use. Visit http://www.dovepress.com/ testimonials.php to read real quotes from published authors. 